A Progress Report to the Nuclear Physics Division of the US Department of Energy for

\title{
Investigation of Rare Particle Production in High Energy Nuclear Collisions
}

(Grant DE-FG03-90ER40571, for the period 12/15/98-12/14/99)

\author{
Henry J. Crawford, Principal Investigator \\ Jon M. Engelage, Co-experimenter \\ University of California, Berkeley \\ Space Sciences Laboratory \\ Berkeley, CA 94720-7450 \\ Recipient Organization: \\ The Regents of the University of California \\ c/o Sponsored Projects Office \\ University of California, Berkeley \\ 336 Sproul Hall \# 5940 \\ Berkeley, CA 94720-5940
}

RECEIVET

JAN 242000

OSTI

Amount of unobligated funds estimated to remain at the end of the current budget period: none.

\section{Summary}

Our program is an investigation of the hadronization process through measurement of rare particle production in high energy nuclear interactions. Such collisions of heavy nuclei provide an environment similar in energy density to the conditions in the Big Bang. We are currently involved in two major experiments to study this environment, E896 at the AGS and STAR at RHIC. We have completed our physics running of E896, a search for the $H$ dibaryon and measurement of hyperon production in AuAu collisions, and are in the process of analyzing the data. We have produced the electronics and software for the STAR trigger and will begin to use these tools to search for anti-nuclei and strange hadrons when RHIC turns on later this year. 


\section{DISCLAIMER}

This report was prepared as an account of work sponsored by an agency of the United States Government. Neither the United States Government nor any agency thereof, nor any of their employees, make any warranty, express or implied, or assumes any legal liability or responsibility for the accuracy, completeness, or usefulness of any information, apparatus, product, or process disclosed, or represents that its use would not infringe privately owned rights. Reference herein to any specific commercial product, process, or service by trade name, trademark, manufacturer, or otherwise does not necessarily constitute or imply its endorsement, recommendation, or favoring by the United States Government or any agency thereof. The views and opinions of authors expressed herein do not necessarily state or reflect those of the United States Government or any agency thereof. 


\section{DISCLAIMER}

Portions of this document may be illegible in electronic image products. Images are produced from the best available original document. 


\section{A. Progress Report}

The University of California Space Sciences group is the lead institution for E896 (CoSpokesman $\mathrm{H}$. Crawford). We have made a first pass through the data at UTA looking for $\mathrm{H}$ candidates based on their decay including a stiff proton track. We have also nearly completed a second pass at UCLA to save all events having a reconstructable neutral vertex. Each of these methods selected $\sim 10 \%$ of the data set. These summary sets are now being analyzed in detail for $\mathrm{H}$ and $\Lambda$ content. We have used the data from our proton run to calibrate the system and are using our $\Lambda$ analysis to tune the $H$ algorithms. A summary of the experiment status as of June 1999 is given in Appendix 1, a paper from the recent Quark Matter Conference.

Our group is also responsible for the design, implementation, and operation of the trigger system for the STAR experiment ( Solenoidal Tracker at RHIC, the Relativistic Heavy Ion Collider) at the Brookhaven National Laboratory (BNL). We have completed fabrication of all trigger components, tested a subset during the recent engineering run, and expect to complete installation in late 1999. STAR will begin study of AuAu interactions at the $100 \mathrm{GeV}$ collider, equivalent to $20 \mathrm{TeV} /$ nucleon in the laboratory, early in 2000 .

\section{A.1. $\mathrm{E896}$}

Experiment E896 at the Brookhaven National Laboratory AGS is a search for the $\mathrm{H}$ dibaryon and for short-lived strange matter, and an investigation of hyperon production in $11 \mathrm{GeV} /$ nucleon AuAu collisions. The $\mathrm{H}$ dibaryon is the lightest example of strange matter, predicted to exist as a 6-quark object (uuddss) within the framework of the MIT bag model (Jaffe 1977). Many experiments have been performed to find the $\mathrm{H}$, but so far no unambiguous signals have been reported (Aoki 90; Belz 96,97; Longacre 98; Stotzer 97). Observations of possible double-hypernuclei events have suggested constraints on the mass of the $\mathrm{H}$ (Aoki 91) but these results are ambiguous. New experiments at the AGS will provide much cleaner investigations of the existence of double hypernuclei, but the analyses of these are still in progress (Rusek 99). Searches for long-lived strange matter have set stringent limits on its production in heavy ion collisions (Beavis 95). We completed our first physics run for E896 in April 1998, a dataset which should contain between 10 and 50 identifiable $\mathrm{H}$ particles if the theoretical predictions are correct (Dover 91). We completed a proton calibration run in September 1998. Preliminary results have been presented at the DNP meetings in Sante Fe and in Atlanta, at the Winter Workshop at Park City, and at Quark Matter 99 which is included here as Appendix I.

E896 is designed to identify an $H$ particle through its decay into either $\Sigma p$ or $\Lambda p \pi$ decay channels. It has limited sensitivity to the $\Lambda \mathrm{n}$ decay channel as well. The experiment consists of two topological signature detectors (a 15 layer Silicon drift array (SDDA) and a 144 plane drift chamber (DDC)) as well as beam vector detectors (BVD) to measure the beam trajectory onto the target, multiplicity detectors (MLT) and exit-charge-detectors (ECD) to determine interaction centrality (multiplicity), and particle identification detectors to verify proton-pion identity through Time-of-Flight (TOF). In addition, a high efficiency neutron 
detector (MUFFINS) is used for redundant identification of the $\Sigma$ through its neutron decay channel. The apparatus as staged in the April 1998 run is shown in Figure 1.

\section{BNL-AGS E896 EXPERIMENTAL LAYOUT}

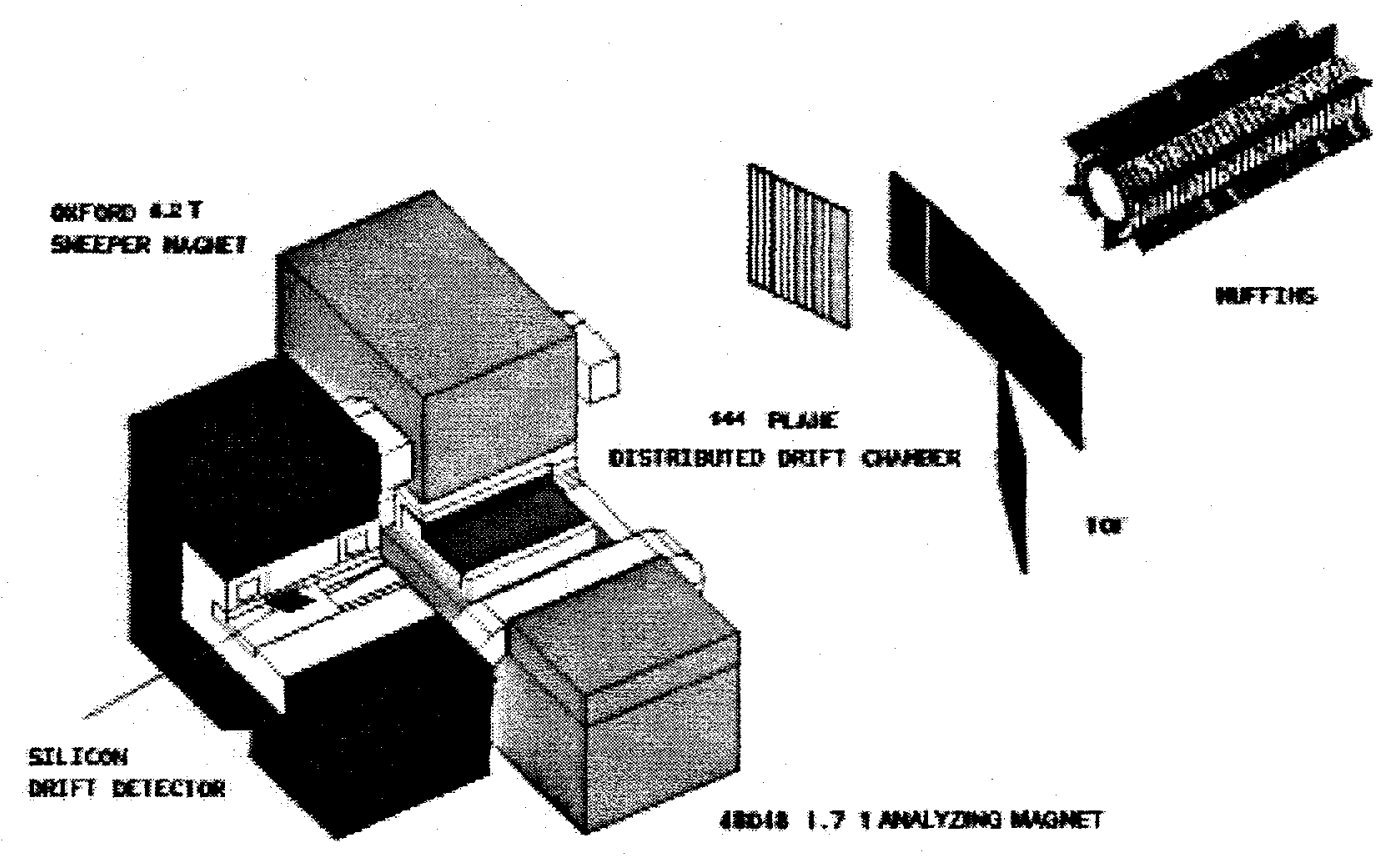

Figure 1: Apparatus for E896 at the AGS.

Data analysis responsibility is divided as follows. The SDDA analysis is primarily the responsibility of Wayne State University, University of Texas (Austin), Ohio State University, and BNL. Rice University is responsible for analysis of the TOF system. University of Catania (Italy) is responsible for the neutron detector (MUFFINS). Carnegie Mellon University and Johns Hopkins/GSFC are responsible for the multiplicity detector (MLT). BNL is responsible for the Beam Vector Detector (BVD). DDC analysis has been performed primarily at UCLA and UTA. Simulations and overall coordination are the responsibility of UC/SSL.

The April 1998 run went extremely well, with the Oxford dipole, the Sweeper, operating at $95 \%$ of its design field or $6.1 \mathrm{~T}$. The 15 layer SDDA performed well in this field and integrated $>5 \times 10^{5}$ central AuAu collisions on tape. The full 144 plane DDC also operated well, integrating $>10^{8}$ central AuAu collisions using much improved electronics. The predicted $\mathrm{H}$ and hyperon signals are shown in Appendix 1 . The difference in dataset size was a result of the fact that the DDC data acquisition was designed for speed, allowing in excess of 1000 events per "spill", while the SDDA system allowed only a few events per spill because each event contained a full non-zero-suppressed readout. 
The SDDA analysis does not overlap with any of the other detectors, relying on $\mathrm{dE} / \mathrm{dx}$ for particle identification and on its hits for tracking in the $6.1 \mathrm{~T}$ field. The drift chamber does not record ionization so it must work with TOF and MUFFINS to complete the particle identification for tracks. Both SDDA and DDC require extensive calibration to sort out tracks correctly, and this is now nearly complete for both. Both require analysis of the beam trajectories and of the centrality selection. We will first discuss the drift chamber analysis, then the silicon detector analysis, and finally the full experiment.

The DDC analysis is expected to require three passes through the full dataset: a first pass to see if a signal stands out, a second pass to improve our efficiency for finding and fitting the signatures, and a final pass to get the best mass resolution and to get the cross sections correct. We have completed the first pass and are $80 \%$ finished with the second pass. We have used two separate approaches to data filtering. The first is performed at UTA and is based on selecting events having a stiff proton that originates within the fiducial volume of the drift chamber. This is a signature of both $\Lambda$ decay and of $H$ decay that can be found quickly with high efficiency. The second approach is performed at UCLA and consists of fitting every track in the chamber. The tracks are then pair-wise compared to see if a) they meet end-point-proximity criteria ${ }^{1}$ or b) they meet distance-of-closest-approach criteria ${ }^{2}$. These two software triggers account for $70 \%$ and $30 \%$ of the selected data respectively.

In September 1998 we had a proton run to provide calibration for our system. We used a Be target to minimize track backgrounds. We employed a $\Lambda$-selecting trigger based on hits in our TOF wall that proved quite effective, allowing us to record events containing $\sim 10^{6} \Lambda$ particles. This dataset provides very clean lambda samples and has been analyzed to complete the calibration of our DDC. Our track fitting error analysis is performed in tdc space because of the magnetic field induced distortion in the drift time vs. distance curve. The drift curve analysis is presented in Figure 2, which shows the effect on the tdc value of the angle at which a track crosses the wire plane. Both the cross section (Blobel 74) and the polarization (Tonse 94) for $\Lambda$ s from $\mathrm{pBe}$ have been measured previously by others, thereby providing a benchmark for our methods.

The simulation effort has a number of goals, including understanding our acceptance, our efficiency, and our backgrounds. To understand the acceptance we have used GEANT to propagate a "white" spectrum through the experiment and into the detectors. The DDC and SDDA lambda acceptance in rapidity $(y)$ vs perpendicular momentum $\left(p_{t}\right)$ coordinates are shown in the Appendix 1. As the acceptance grid was populated in CPU intensive calculations at both UCLA and NERSC ${ }^{3}$ each "accepted" particle was saved so that we can use them to test improvements in our reconstruction code as it evolves. We have produced a full simulation chain and used the measured resolution of the detectors to embed $\Lambda$ and $H$ particles in a subset of the real data. These embedded events provide the basis for our finding and fitting efficiencies.

\footnotetext{
${ }^{1} \delta \mathrm{x}<0.5 \mathrm{~cm}, \delta \mathrm{y}<2.0 \mathrm{~cm}, \delta \mathrm{z}<2.0 \mathrm{~cm}$

${ }^{2} \delta x<10 \mathrm{~cm}$ and $\delta y<5 \mathrm{~cm}$ and $\delta r_{x}<1 \mathrm{~mm}$ and $\delta r_{y}<5 \mathrm{~mm}$

${ }^{3}$ National Energy Research Supercomputer Center at Lawrence Berkeley National Laboratory.
} 


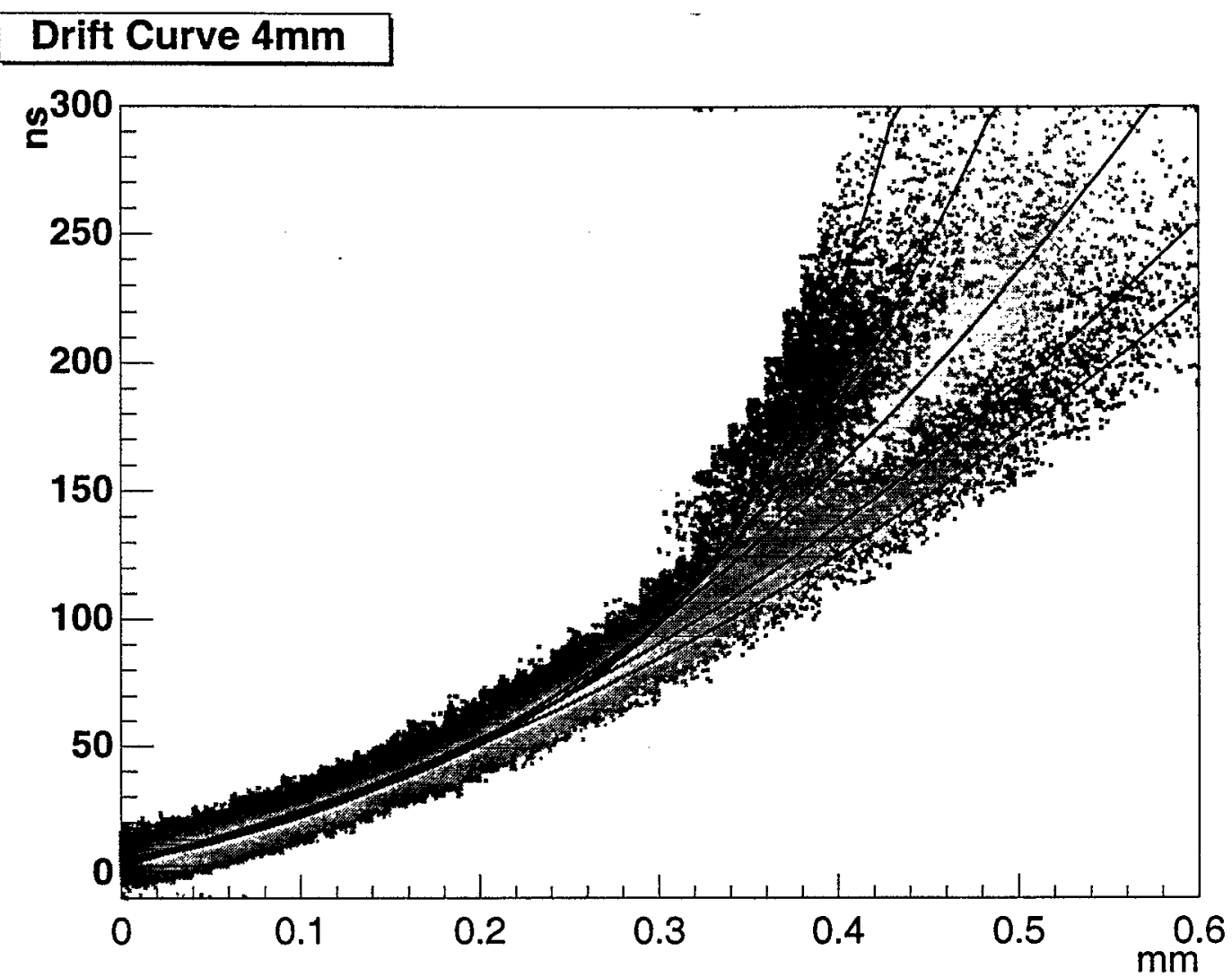

Figure 2: Drift curve analysis showing multiple curves required as a result of magnetic distortions in time-vs-distance function.

We can reconstruct the invarient mass of each vertex with a variety of assumptions concerning the daughter particle's identification. We first cut on vertices that have a net momentum pointing back to the target. The target spot is measured by the BVD for each accepted beam particle. It is also measured by the spot onto which the neutral vertices project, since most of these originate in the target. The spot is also measured using the SDDA to point back to the target for all tracks in an event. Cutting on the target location leads to a mass distribution for $\mathrm{K}$ and $\Lambda$ as shown in Appendix 1. The target-pointing cut is one of the most powerful background rejection cuts in the $\mathrm{H}$ analysis.

The SDDA zero suppressed data has been distributed from UTA to WSU and OSU for analysis - tracking, particle identification, cross section calculations. The SDDA analysis has already resulted in two $\mathrm{Ph}$. $\mathrm{D}$ theses, and we expect two more in FY00. Vertex code has been developed to search for $\Lambda, \Xi$, and $H$ particles. An example of the current status of the $\Lambda$ analysis is shown in Appendix 1, a $\Lambda$ mass histogram. PID Based on $\mathrm{dE} / \mathrm{dx}$ as shown in Appendix 1 clearly indicates protons and heavier baryons, allowing us to check deuteron coalescence parameters, especially as they apply to $H$ formation through $l \Lambda \Lambda$ coalescence.

\section{A.2. STAR}


The goal of the STAR experiment is to uncover evidence for the formation of a plasma made up of essentially free quarks and gluons such as was expected to prevail in the early universe just prior to hadron formation. The final state signature of this plasma formation is not clearly understood, however. The process of initial hadronization from the plasma may well be masked by subsequent interactions among the produced hadrons in an expanding and cooling hadron gas phase. A possible unambiguous signature of the existence of the plasma phase may be the formation of a large (baryon number $A>10$ ) multi-quark nugget of strange matter. Or, indication of its existence may come in the form of non-statistical fluctuations in the phase space populations of particles. We have been charged with providing a trigger system flexible enough to encompass a broad range of possible signature states, allowing selection of any combination of particles in the final state distribution.

Our solution for the STAR trigger is a multi-level, modular, pipelined system relying on high speed programmable gate arrays followed by fast standard processor farms. This is shown schematically in Figure 3. There are three levels that make use of information from the fast trigger detectors, with the timing set by response time for the STAR Time Projection Chamber (TPC) : $1 \mu \mathrm{s}$ to open its grid, $100 \mu \mathrm{s}$ to collect its samples, and $5 \mathrm{~ms}$ to digitize and store the data.. The first is Level 0 , which receives data from the fast detectors and issues triggers. The fast detectors include the Central Trigger Barrel (CTB), the MultiWire proportionsal Chamber (MWC), the Zero Degree calorimeter, and, soon, the Electromagnetic Calorimeter (EMC). Level 0 is followed by Levels $1 \& 2$, which are two sets of veto processing units that look at the triggered events in more detail and decide whether to abort them or pass them on to the next level of analysis. The major difference between Level 1 and Level 2 is the available time and granularity of the input data. These first three trigger levels are implemented in VME, using a mix of custom designed boards and commercial CPUs. Level 0 consists of three types of boards: a set of digitizer (ADC) boards for the fast detectors, a tree of data storage and manipulation (DSM) boards, and a trigger control unit (TCU). All of these electronics boards were designed and fabricated by UCB/SSL working closely with LBL engineers.

The CTB consists of 240 scintillator slats viewed by photo-multiplier tubes (PMTs) . It was produced at Rice University and installed at STAR in Winter 1999. This included installation of the PMT bases which were designed, prototyped, and produced by the UC/SSL group. Output from the PMTs is sent to the ADC boards where they are digitized each RHIC crossing. To aid in Cosmic Ray (CR) rejection the signals are each checked against a local threshold to determine whether they originated at the correct time. Our CR background is expected to be a few $\mathrm{kHz}$ producing low multiplicity coincidences. These events are distinguished from peripheral $\mathrm{AuAu}$ interactions by their geometrical and time distributions, and can be actively ignored at Level0. All test results are catalogued on the $\mathrm{WWW}^{4}$.

The MWC system counts hits on individual anode wires of the TPC and makes these available at Level0 for the initial trigger decision. This system was designed and fabricated primarily by the LBL Nuclear Science Division in consultation with our group. We have tested all of its major components and expect to install it with the other trigger electronics in September 1999.

${ }^{4}$ On the STAR AFS web area 


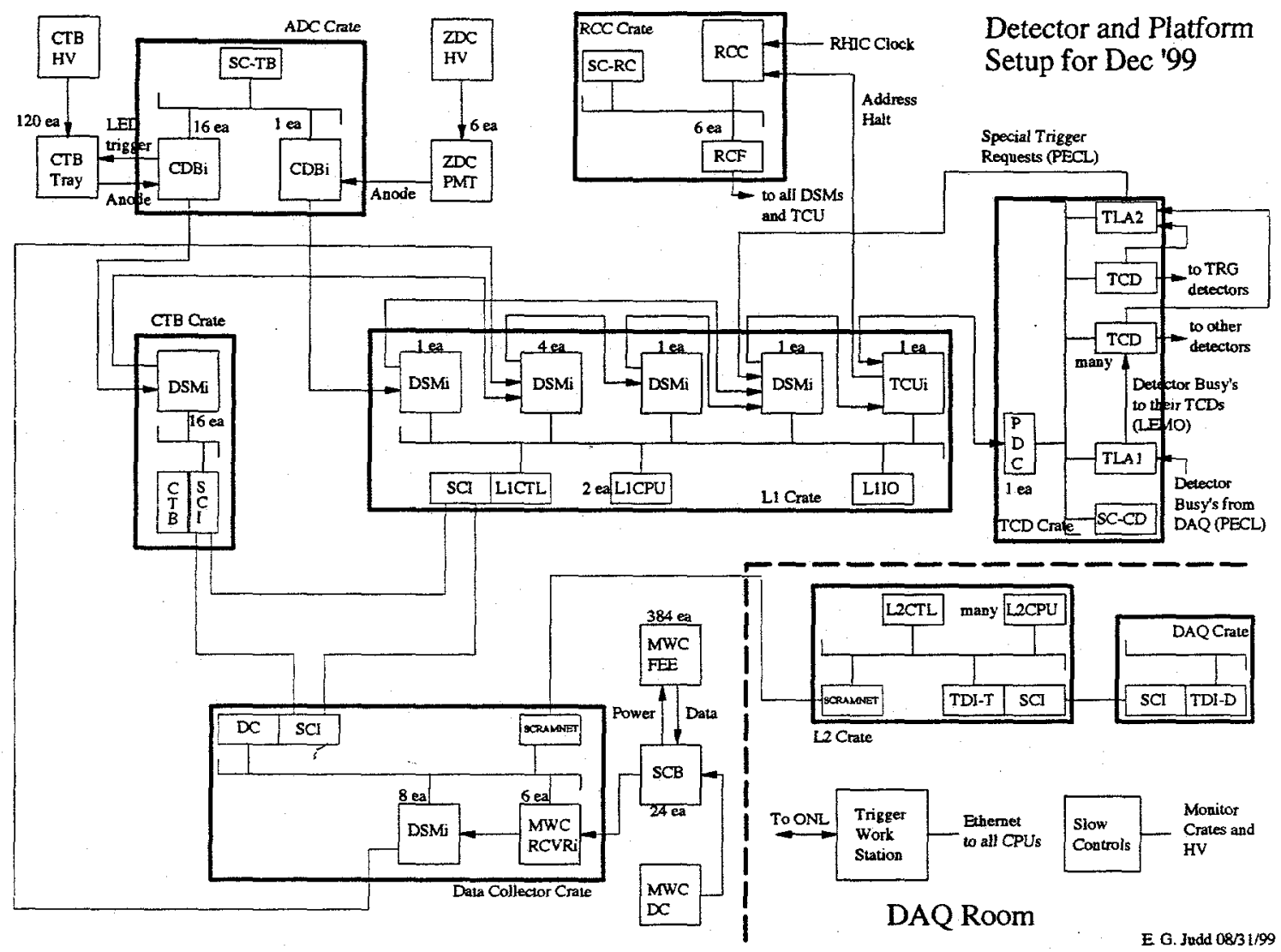

Figure 3: The crate layout for the STAR trigger in December 1999.

The Zero Degree Calorimeters sit at either end of the STAR intersection region at the crotch of the $\mathrm{dx}$ magnets in the Wide Angle Hall (WAH). These are designed to resolve individual neutrons liberated in the fragmentation region, acting as a further check on the centrality of the collisions. There are two ZDCs, one at the East end and one at the West end of the WAH. These have been installed and tested during the summer engineering run. The ZDCs use the same ADC board and fast logic used by the CTB.

The DSM boards constitute data receivers and memory for the digital signals from the three fast trigger detectors. These boards will also be used for the EMC tower signals when that detector is installed. Each board has 128 input bits, 32 output bits, a $64 \mathrm{~K}$ memory and a field programmable gate array (FPGA) capable of computing simple sums, minima, et cetera. We have produced and tested 40 DSM boards for use in the Level 0 trigger. All tests are logged on the web.

The Trigger Control Unit accepts data from the DSM tree and compares the bits with a prescale value to determine if an interaction of interest occurred. When such an interaction occurs, the TCU issues a token for the event and passes that token and information concerning the event handling to the Trigger-Clock Distribution (TCD) network. The TCDs subsequently send this information to the detector systems (TPC, EMC, etc.). Once a token is assigned to an event it stays with that data set until the event is written to tape by 
the data acquisition group (DAQ) or aborted. The token is then returned to the pool. The number of tokens is chosen based on the amount of resources available in the trigger system so no event gets stuck waiting to be processed. We have produced and tested three TCU modules. All tests are logged on the web.

The RHIC Clock and Control (RCC) board, and the RHIC Clock Fan-out (RCF) boards, receive the clock from RHIC and distribute it to the DSM and TCU boards. The TCU then distributes this clock to the rest of the STAR electronics system through the TCD. The RCC and RCF boards were designed, prototyped, produced and tested in FY99.

As part of the trigger our group is responsible for connections between trigger and experiment control efforts, interfacing to both the Online and Slow Controls groups. We have implemented and tested the high voltage control and monitor scheme, and have established all slow control connections necessary to maintain our hardware. We have exercised basic socket functionality for the connection to Online, and have developed our internal trigger control code to communicate with the Online system.

RHIC had an engineering run in June and July of 1999 to bring the accelerator and detector online. For this run we had installed the CTB with its high voltage and all cabling, two of the ADC boards, a TCU, the internal trigger network and processors for each trigger level. We used the CTB to form cosmic ray and interaction triggers. We used the TCU to issue events and respond to detector and DAQ generated BUSY signals. Data were simulated for each DSM, packed into events and shipped across the SCRAMNET fiber network from the WAH platform to the DAQ room, packaged and sent to the Trigger-Daq-Interface (TDI) (see Figure 3). While all aspects of the trigger were tested, the TDI was not employed during the run since we ran much simpler handshake protocols to couple the trigger and DAQ systems for tests and data taking. We succeeded in taking thousands of CR Triggers and "interactions" triggers during this test run, exercising the flexible software configuration for trigger selection.

Most of the group's efforts in FY99 have been directed at production and testing of the STAR trigger system, a much larger fraction than we had originally estimated. This is due in part to the ADC board design and fabrication task that was not originally planned for our group. All of the testing and debugging of the ADC, DSM, TCU, and RCC boards working together also took much more effort than planned.

\section{B. Expected Progress in FY00}

\section{B.1. $\mathrm{E} 896$}

Our primary goal for Fy00 is to complete analysis and publication of the data for both the DDC and SDDA. If we have strong evidence for the existence of the $\mathrm{H}$ we intend to request time for a follow-up run for E896. We would expect this to take place in FY01, after we have reestablished the system for data taking. Note that E896 was originally approved for 1000 data taking hours, but that the short (4 week) run in April 1998 and the shorter ( 2 weeks - caused by magnet problems) engineering run in Jan 1997 have produced less than 500 hours of data so far. 
The bulk of the DDC analysis is now being performed at UCLA, UCB, and BNL. The modules of the analysis package have been developed and are being fine tuned for efficiency and resolution.

Our $\mathrm{H}$ finding code is presently running at only $1-2 \%$ overall reconstruction efficiency, and our highest priority is to improve this to the $10 \%$ level on which we based our original yield estimates. We have developed two approaches to the $\Sigma$ p analysis, distinguished by their analysis of the kink in the negative track caused by the $\Sigma->\pi$ decay. The first is called the small-kink analysis which takes hits on the negative track and starts at both ends to determine whether the collection of points is better described as two intersecting tracks or as a single track. The second is the large kink analysis which assumes that the original vertex was correctly located and that a negative third track intersected the disappearance point of the negative partner. This code development and tuning is expected to take another 3-4 months of effort.

After perfecting the kink finding code we need to apply the topological refit code to the full $\mathrm{H}$ decay. Refining this code module is expected to proceed in parallel with the kink code development. When these code segments are ready we will run both of our summary data sets through them, the UTA set based on the stiff proton, and the UCLA set based on the neutral vertex. The pass through the data is expected to take 1-2 weeks at most.

When our signal has been established, assuming it is, we will then repeat our background analysis to eliminate all potential background candidates from the set. This analysis is expected to require another 2 months of effort.

Once we have fully tuned the tracking code, we can select a subset of the $\Lambda \mathrm{s}$ that do not point to the target as indicating possible candidates for $H$ decay through the $\Lambda \mathrm{p} \pi$ channel. Most of our effort will be concentrated on the $\Sigma$ p channel until we have that analysis completed. We will then turn our attention to the $\Lambda \mathrm{p} \pi$ decay which searches events having two reconstructable neutral vertices. If the momentum vector of the $\Lambda$ vertex points to the $p \pi$ vertex, we have a candidate for an $H->p \pi \Lambda$.

In addition to the $\mathrm{H}$ analysis, we intend to complete the $\Lambda$ polarization analysis early in Fy00. This work is done almost exclusively at UCLA but is an integral part of the E896 program. We also intend to analyze the dataset for $\Lambda \Lambda$ correlations. We expect to have $\sim 2 \mathrm{k}$ events having two reconstructed $\Lambda$ particles and expect to be able to measure the relative momentum distribution for these. This effort will be concentrated at UCB for the DDC datasets.

The kink finder code being developed for DDC analysis will be applicable to the SDDA H analysis to be done primarily at UTA. The approach will use the SDDA hits to investigate goodness-of-fit for two-track and one-track hypotheses for small kinks and for intersecting tracks for larger kink angles.

At least two other SDDA analyses will be completed in FY00, the analysis of $\Xi$ and $\Lambda \bar{\Lambda}$ signals. These will be performed primarily at OSU and WSU. 


\section{B.2. STAR}

\section{B.2.1 Electronics}

All of the electronics for the baseline trigger is now involved in tests at LBL prior to installation in September 1999 at BNL. Each module has undergone an individual test, and the scripts for these tests are now standardized for each type of module. Each script provides a log file documenting the test results. The LBL test includes all cabling to complete the system. The modules will all be run under the normal trigger operating system (L0-L1-L2) before shipment.

Once at BNL, the installation is expected to take 1-2 weeks for full checkout. Each module will be tested individually and in system operation using the scripts developed at LBL. We intend to produce operator's manuals for each of the modules and for the trigger system as a whole during FY00. A major portion of the effort in Fall 99 will be integration of the trigger with the Online experiment control system. While we have all parts of the trigger software working stand-alone, we need to take commands from the Online processes to coordinate STAR operation. Included here will be the conversion of all of our configuration files into database objects to conform with STAR software guidelines.

The trigger system has already been integrated with the TPC during the engineering run. While this connection can improve, the basic operating functions have been tested. We need to integrate with the other detector subsystems, which we expect to accomplish as each is installed. We have already connected to the RICH detector and expect to achieve working calibration triggers with them before the run begins.

We have agreed to fabricate the Level 0 electronics for the EMC. This will involve production, testing, and installation of 35 DSM modules for use in the trigger. Each tower will provide 12 bits indicating the shower sum and the highest sub-tower signal. These bits will be used in an EMC DSM tree just like the CTB and MWC trees, providing a level 0 indication of jets. We have begun an MOU with LBL and WSU concerning this effort which is included as an appendix here.

We have signed an MOU with BNL concerning our role in trigger operation which is included as another appendix. The intent is for our UCB/SSL group to act as the trigger experts for hardware and software development and for trouble-shooting throughout the life of STAR.

\section{B.2.2 Software}

As the RHIC turn-on approaches, the SSL group is beginning to concentrate its physics analysis efforts in the exotic particle subgroup within the STAR Spectra physics working group. This subgroup is a natural extension of the rare particle searches that the group has been involved with over the last decade at the AGS. We will work on all trigger levels, including the Level 3 tracking trigger, to improve our selection criteria for events having embedded strangeness and anti-nuclei. The benefit of increasing the signal to noise ratio at the trigger level can not be underestimated.

We expect to develop an ionization/ curvature trigger for application at Level 3 in selection of strange matter and anti-nuclei signals. The STAR TPC provides a measurement of the 
energy deposition $(\mathrm{dE} / \mathrm{dx})$ for the various tracks in an event. Coupled with the momentum determined by the curvature of the tracks in the magnetic field provided by the solenoid, tracks from normal matter will be centered on one of the prominent Bethe-Bloch curves. Heavy strange matter candidates will not fall on those curves, but are expected to concentrate in the upper right comer of such a plot. Heavy anti-nuclei $(\mathrm{Z}=2$ or 3$)$ will be well above the background curves on the negative charge side.

Our immediate trigger emphasis will be on detecting heavy anti-nuclei. Using simple coalescence arguments and parameters measured in central $\mathrm{AuAu}$ and $\mathrm{PbPb}$ collisions we find that RHIC may produce as many as $3 \times 10^{4}$ anti-alpha particles per central collision. Although experiments at AGS, CERN, and DUBNA have observed anti-deuterons, antitritons, and anti- $\mathrm{He}^{3}$, no one has ever seen an anti- $\mathrm{He}^{4}$. Initial simulations of this show that we can expect $\sim 10 \overline{\mathrm{p}}$ per CC identifiable in STAR based on $\mathrm{dE} / \mathrm{dx}$ and rigidity $(\mathrm{R})$ measurement. Assuming that our efficiency for $\bar{\alpha}$ is the same as for $\bar{p}$ we can then expect to see $\sim 2 \cdot 10^{-5} \bar{\alpha}$ per CC in STAR. This is a conservative estimate because our efficiency for finding the $\bar{\alpha}$ in the $d E / d x$ vs. R plot is much better than for $\bar{p}$ because there is much less background. In fact, we believe this is a triggerable signature at level 3 in STAR, perhaps allowing us to reach $\bar{A}=6$. Measurement of anti-nuclei provide a tool for investigation the hadronization length for anti-nucleons. 


\section{REFERENCES}

S.Aoki et al., Phys.Rev.Lett. 65, 1729 (1990)

S.Aoki et al., Prog.Theor.Phys.85, 1287 (1991).

D.Beavis et al. Phys.Rev.Lett. 75, 3078 (1995a)

V.Blobel et al. Nuc. Phys. B69 454 (1974)

J. Belz et al, Phys. Rev. Lett. 76, 3277 (1996)

J. Belz et al, Phys. Rev. C56, 1164 (1997)

C.B.Dover, Nuovo Cimento A 102, 521 (1991)

R. L. Jaffe, Phys. Rev. Lett. 38, 195 (1977)

R.Longacre, private communication.

A.Rusek, AGS User's Meeting, July 1999.

S.Tonse, PhD.Thesis, Rice University, 1994.

R.W.Stotzer et al., Phys.Rev.Lett. 78, 3646 (1997).

\section{BIBLIOGRAPHY}

\section{$\underline{\text { Refereed Publications }}$}

"Particle Production at High Baryon Density in Central Au+Au Collisions at $11.6 \mathrm{~A}$ GeV/c," L.Ahle and the E866 Collaboration, Phys.Rev.C 57, R466 (1998)

"Au+Au Reactions at the AGS: Experiments E866 and E917," C.A.Ogilvie and the E866 Collaboration, Nucl.Phys. A A638, 57c (1998)

"Centrality and Collision System Dependence of Antiproton Production from p+A to Au+Au Collisions at AGS Energies," H.Sako and the E866 Collaboration, Nucl.Phys.A A638, 427 (1998)

"Antiproton Production in Au+Au Collisions at 11.7A GeV/c," L.Ahle and the E866 Collaboration, Phys.Rev.Lett. 81, 2650 (1998)

"Kaon Production in $\mathrm{Au}+\mathrm{Au}$ Collisions at 11.6A GeV/c," L.Ahle and the E866 Collaboration, Phys.Rev.C 58, 3523 (1998)

"Neutron Production in Coincidence with Fragments from the ${ }^{40} \mathrm{Ca}+\mathrm{H}$ Reaction at $E_{\mathrm{ab}}=357 \mathrm{~A}$ and 565A MeV," C. Tuve and the TRANSPORT Collaboration, Phys.Rev.C 59,1 (1999) 
"Simultaneous Multiplicity and Forward Energy Characterization of Particle Spectra in $\mathrm{Au}$ $+\mathrm{Au}$ Collisions at 11.6A GeV/c," L. Ahle and the E802 Collaboration, Phys.Rev.C 59,2173 (1999)

\section{Conference Proceedings and Other Publications}

"Prediction and Detection of Ultra High Energy Neutrino Bursts," M. Roy, H.J. Crawford, A. Trattner, to be published in DPF99 (American Physical Society meeting), Astroph/9903231, (Los Angeles) (1999)

"Strange Baryon and H-Dibaryon Measurements in Au-Au Collisions at the AGS," R. Bellwied for the E896 Collaboration, Advances in Nuclear Dynamics 4, Proceedings of the 15th Winter Workshop on Nuclear Dynamics, Feb. 1999, Park City, Utah, USA

"Strange and Multi-strange Baryon Measurement in $\mathrm{Au}+\mathrm{Au}$ Collisions at $11.6 \mathrm{~A}(\mathrm{GeV} / \mathrm{c})$ With the Silicon Drift Detector Array From the AGS Experiment E896," Quark Matter 1999, Torino, Italy, June (1999)

"First Results From the $\mathrm{H}_{0}$ Di-baryon Search and Hyperon Production Measurements by the AGS Experiment 896," Quark Matter 1999, Torino, Italy, June (1999)

Appendices

1. Quark Matter summary of E896

2. Memo of Understanding between SSL and BNL for trigger operation

3. Memo of Understanding between SSL and LBL for fabrication of EMC electronics 
First results from the $H_{0}$ di-baryon search and hyperon production measurements by the AGS Experiment 896.

H. Caines ${ }^{a}$, S. Albergo ${ }^{e}$, R. Bellwied ${ }^{l}$, M. Bennett $^{j}$, D. Boemi ${ }^{e}$, B. Bonner ${ }^{i}$, Z. Caccia ${ }^{e}$, W. Christie ${ }^{b}$, S. Costa ${ }^{e}$, H. Crawford ${ }^{j}$, M. Cronqvist ${ }^{j}$, R. Debbe $^{b}$, J. Engelage $^{j}$, I. Flores ${ }^{j}$, L. Greiner ${ }^{j}$, T. Hallman ${ }^{b}$, G. Hoffman ${ }^{k}$, H. Huang ${ }^{c}$, T.J. Humanic ${ }^{a}$, G. Igo ${ }^{c}$,

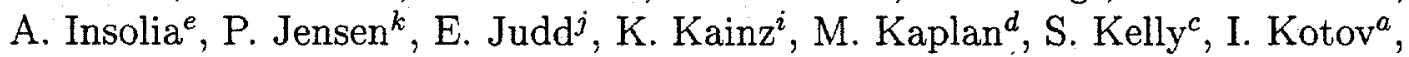
G. Kunde ${ }^{m}$, P. Lindstrom ${ }^{g}$, T. Ljubicic ${ }^{b}$, W. Llope ${ }^{i}$, G. Lo Curto ${ }^{a}$, R. Longacre ${ }^{b}$, D. Lynn ${ }^{b}$, L. Madansky ${ }^{f}$, N. Mahzeh ${ }^{l}$, Z. Milosevich ${ }^{d}$, J. Mitchell ${ }^{b}$, J. Mitchell ${ }^{h}$, S. Nehmeh ${ }^{l}$, C. Nociforo ${ }^{e}$, S. Paganis ${ }^{k}$, S.U. Pandey ${ }^{l}$, R. Potenza ${ }^{e}$, E. Platner ${ }^{i}$, P. Riley ${ }^{k}$, D. Russ ${ }^{d}$, G. Russo ${ }^{e}$, A. Saulys ${ }^{b}$, J. Schambach ${ }^{k}$, J. Sheen ${ }^{l}$, C. Stokley ${ }^{i}$,

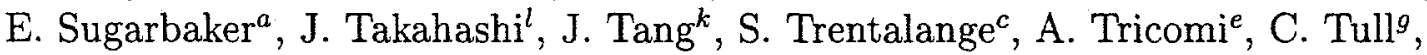
C. Tuve ${ }^{e}, J$. Whitfield ${ }^{d}$, K. Wilson ${ }^{l}$

The E896 Collaboration

${ }^{a}$ The Ohio State University, Columbus, Ohio 43210

${ }^{b}$ Brookhaven National Laboratory, Upton, New York 11973

'University of California, Los Angeles, California 90095

${ }^{d}$ Carnegie Mellon University, Pittsburgh, Pennsylvania 15213

eUniversity of Catania, 95129 Catania, Italy

$f$ Johns Hopkins University, Baltimore, Maryland 21218

${ }^{g}$ Lawrence Berkeley Laboratory, Berkley, California 94720

${ }^{h}$ NASA - Goddard Space Flight Center, Greenbelt, Maryland 20771

${ }^{i}$ Rice University, Houston, Texas 77251

${ }^{j}$ Space Sciences Laboratory, Lawrence Berkley Laboratory, Berkley, California 94720

${ }^{k}$ University of Texas, Austin, Texas 78712

${ }^{l}$ Wayne State University, Detroit, Michigan 48201

${ }^{m}$ Yale University, New Haven, Conneticut 8124

The AGS Experiment 896 was designed to study strangeness production in $\mathrm{Au}-\mathrm{Au}$ collisions at $11.6 \mathrm{~A} \mathrm{GeV} / \mathrm{c}$, in particular the formation of a six-quark di-baryon the $H_{0}$. Heavy ion collisions provide favorable conditions for the $H_{0}$ formation either via coalescence of two $\Lambda$ particles (owing to the large $\Lambda$ production cross section) or direct production from the possible formation of a quark-gluon plasma. E896 also measured strange meson and baryon distributions from mid-rapidity. Preliminary results from this experiment are presented as well as details of the expected sensitivity for the $H_{0}$ search. 


\section{INTRODUCTION}

The $H_{0}$ di-baryon (a six-quark state of uuddss) was first predicted by R.Jaffe [1] over twenty years ago. Many experiments have since searched for this elusive meta-stable strangelet state e.g. E810 [2], E888 [3], E836 [4] but no conclusive proof has yet been discovered. (See [5] for an overview summary of the search for the $H_{0}$.)

The large production of $\Lambda$ 's in Heavy ion collisions means that there should be a correspondingly high production of di- $\Lambda$ 's from simple coalescence arguments [6]. If the $H_{0}$ is more deeply bound than a coalescing $\Lambda \Lambda$ pair they may spontaneously decay into an $H_{0}$. This process allows for the creation of the strangelet without the need for a QGP formation.

Di- $\Lambda$ events are of interest in the case that the $M_{H 0}>2 M_{\Lambda}$ where the $H_{0}$ will manifest itself as a broad resonance in the invariant mass plot. The di- $\Lambda$ events will also be used to determine an upper limit for the production rate of the $H_{0}$ di-baryon via coalescence per produced di- $\Lambda$.

E896 was optimized to search for the $H_{0}$ using two complementary tracking detectors, a Distributed Drift Chamber (DDC) and a Silicon Drift Detector Array (SDDA). The DDC had a large sensitivity to detect the $H_{0}$ decay modes $H_{0} \rightarrow \Sigma^{-} p \rightarrow p n \pi^{-}$and $H_{0} \rightarrow \Lambda p \pi^{-}$ over a wide range of lifetimes, while the SDDA was ideally positioned for detecting the $H_{0}$, with a $c \tau \sim 4 \mathrm{~cm}$, at the low end of the DDC's sensitivity range.

The SDDA also had a good acceptance for measuring neutral and charged strange particle production, the $\Lambda, \bar{\Lambda}$ and $\Xi^{-}$, around mid-rapidity via their decay products. These results will complement the hyperon measurements already reported by previous SPS and AGS experiments [7]. It is also intended to use the good $\mathrm{dE} / \mathrm{dx}$ resolution obtainable from the SDDA to measure $\bar{p}$ production and investigate the $\bar{\Lambda} / \bar{p}$ ratio. Recent results both at the AGS [8] and SPS [9] have shown unusual behavior in this ratio and E896 will be able to supply an alternative statistically significant direct measurement.

The Distributed Drift Chamber (DDC) identifies $\Lambda$ 's, $\bar{\Lambda}$ 's and $K_{s}^{0}$ 's at high rapidity and low $p_{T}$. The differing acceptances of the SDDA and DDC combined with the symmetry of the collision means that E896 will measure the production of $\Lambda$ 's over virtually the whole rapidity region.

\section{THE EXPERIMENT 896}

The design of E896 can be seen schematically in Fig. 1. The DDC consists of 144 planes with $\sim 8000$ channels and an active volume of $120 \mathrm{~cm} \times 67.5 \mathrm{~cm} \times 20 \mathrm{~cm}$. It was located $1.3 \mathrm{~m}$ downstream of the target in a $1.7 \mathrm{~T}$ analyzing magnet. The SDDA, positioned in the $6.2 \mathrm{~T}$ sweeper magnet approximately $10 \mathrm{~cm}$ downstream of the target, consisted of 15 planes of silicon drift detectors [10]. Each plane of the SDDA was formed by a $6.3 \mathrm{~cm} \mathrm{x}$ $6.3 \mathrm{~cm} \times 300 \mu \mathrm{m} \mathrm{n}$-type silicon wafer. The SDDA was a proto-type of the technology to be used by the STAR experiment [11] in the Silicon Vertex Tracker (SVT).

Although the $H_{0}$ can be identified unambiguously using a constrained fit to the unique topology characteristics of the $H_{0} \rightarrow \Sigma^{-} p$ decay, redundant particle identification will be provided by using the MUlti-Functional Neutron Spectrometer (MUFFINS)[12] to detect the neutron from the subsequent $\Sigma^{-}$decay and the Time-Of-Flight (TOF) walls to aid the definition of the charged tracks reconstructed in the DDC. 


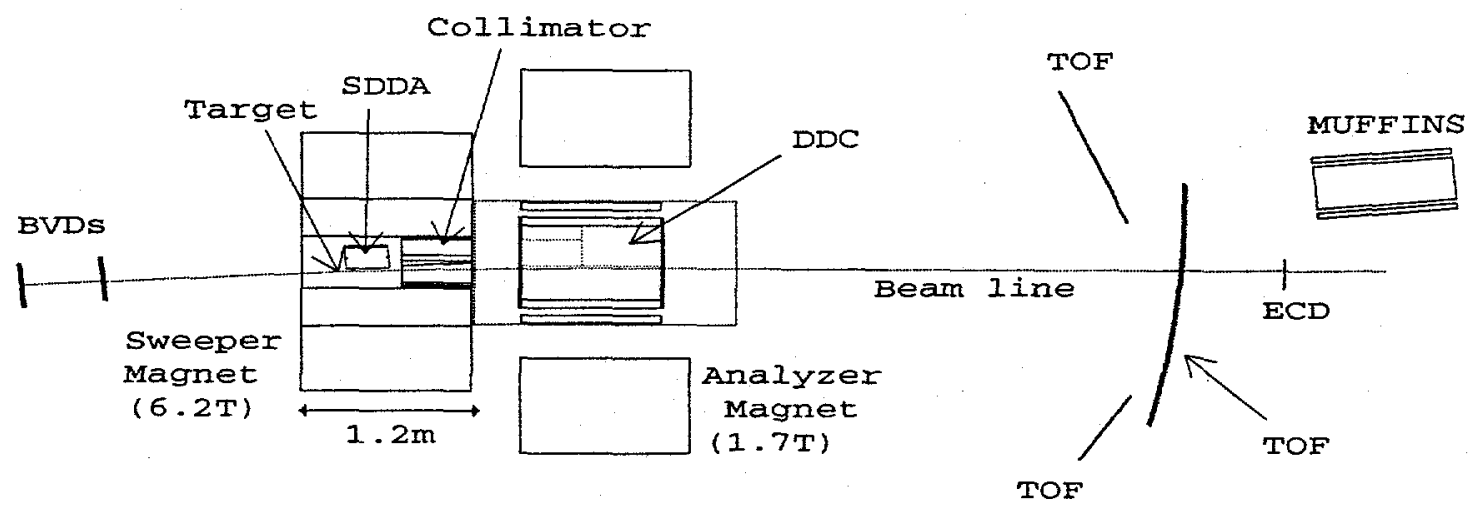

Figure 1. The experimental setup.

Two beam vertexing detectors (BVDs) were used for locating the primary vertex position and a forward multiplicity array determined the centrality of the collision for triggering.

\section{THE RESULTS}

During the April 1998 run 100 million central events were recorded by the DDC and 680,000 central events by the SDDA. Since silicon drift detectors are slow detectors the SDDA was operated using a $1 \mathrm{~Hz}$ data acquisition system, much slower than that of the DDC.

From these data it is predicted, from the DDC acceptance and reconstruction efficiency, that it will be possible to reconstruct $\sim 450,000 \Lambda$ 's, 45,000 $K_{s}^{0}$ 's and $200 H_{0}$ 's. A similar calculation shows that the SDDA data should yield $\sim 200,000 \Lambda$ 's, $400 \bar{\Lambda}$ 's and $500 \Xi$ 's from their data set. The acceptance for the $\Lambda$ 's in the SDDA and DDC is shown in Fig. 2.

The clean environment created by the sweeper field and collimator, on average there are less than 10 tracks in the DDC per event, means that the track reconstruction efficiency in the DDC is very high. Neutral decays occuring within the DDC can be cleanly reconstructed with very little background (Fig. 7). This is to be compared to the higher track density observed in the SDDA of $\sim 60$ particles per event [13].

\subsection{The SDDA results}

Presented here are preliminary results for primary proton and $\Lambda$ reconstruction in the SDDA; for more details on the current progress of SDDA analysis see [13].

The position, and hence momentum, resolution is influenced by external factors, such as the high magnetic field, ambient temperature and sampling frequency. The accuracy of the calibrations, in particular the velocity profile of each detector also have an effect. Bench tests have proven that these detectors are capable of a position resolution of the order of $20 \mu \mathrm{m}$ [14]. Currently the SDDA calibrations show a slightly larger resolution but progress is still being made in this area.

The energy deposited per unit length $(\mathrm{dE} / \mathrm{dx})$ vs momentum plot for positively charged 


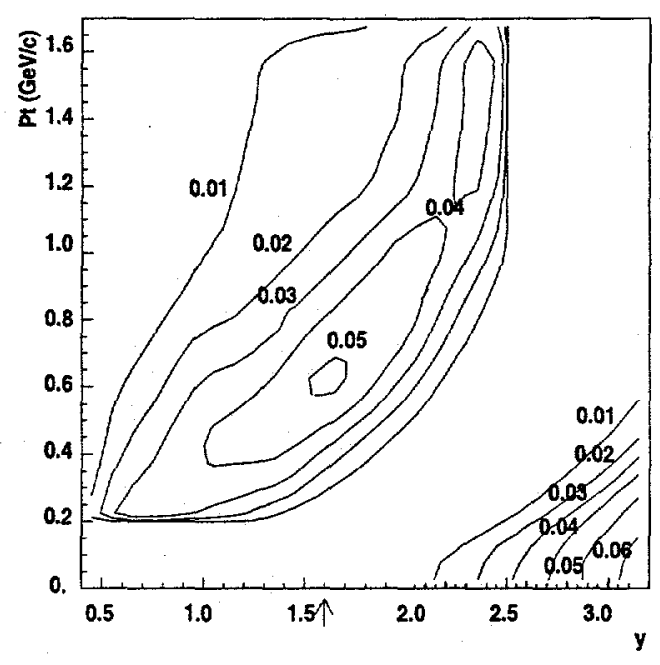

Figure 2. $\Lambda$ acceptance in the SDDA and DDC as a function of $p_{T}$ and rapidity. The contours are labelled with the fractional acceptance and the arrow indicates mid-rapidity.

particles reconstructed in the SDDA for 1000 events can be seen in Fig. 3. A clear proton band can be seen emerging below a momentum of $\sim 0.7 \mathrm{GeV}$. Improving the statistics will allow the clean identification of deuterons which can also be observed in this plot. When the whole data set has been reconstructed it is expected that a positive identification of $\bar{p}$ 's will be possible from a similar plot for negatively charged particles. Fig. 4 compares the $p_{T}$ distribution for identified protons to that expected from Monte-Carlo simulations. It can be seen that $\sim 70 \%$ of the expected protons, those with total momentum $<0.7 \mathrm{GeV}$, are recovered. We currently calculate an overall reconstruction efficiency for primary tracks of $>85 \%$. Work is in progress to try to improve the $\mathrm{dE} / \mathrm{dx}$ calibration and thus increase the separation of the bands allowing the identification of protons to higher total momentum.

Fig. 5 shows the invariant mass distribution for $\Lambda$ 's in the SDDA. 10,000 central events have been reconstructed to produce this distribution. A clear peak can be seen around the nominal $\Lambda$ mass. The solid histogram represents the signal reconstructed from mixed events and undergoing identical cuts. Due to the imperfect calibration of some parts of the SDDA at this time only a portion of the detector is used in the $\Lambda$ reconstruction. This results in a reduced acceptance and reconstruction efficiency to that shown in Fig. 2 and assumed when calculating the yield of $\Lambda$ 's from the total data sample. It is expected that the whole detector will ultimately be used and hence the $\Lambda$ yield per event will be enhanced.

\subsection{The DDC $\Lambda$ and $K_{s}^{0}$ results}

Fig. 6 shows the invariant mass distributions for the $\Lambda$ and $K_{s}^{0} .15$ million events have been reconstructed in these plots with the requirement that the reconstructed v0 occur 


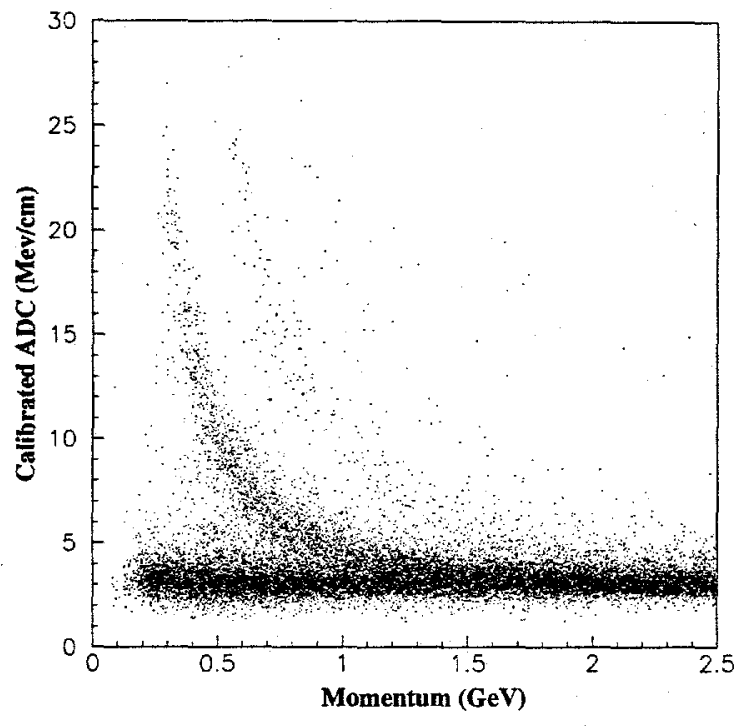

Figure 3. $\mathrm{dE} / \mathrm{dx}$ for positively charged tracks in the SDDA for $\sim 1000$ events.

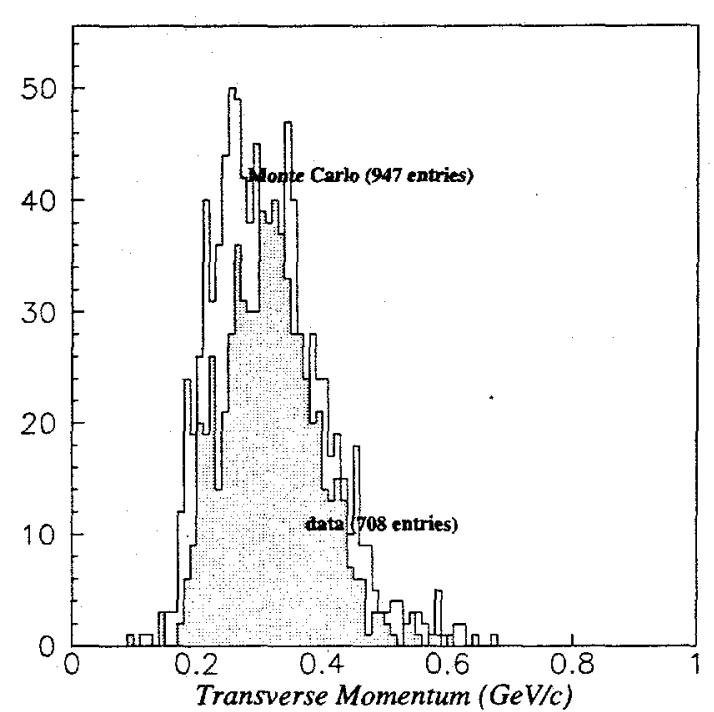

Figure 4. The $p_{T}$ distribution for identified protons from the SDDA (solid plot) compared to Monte-Carlo simulation for an identical number of events. Approximately $70 \%$ of the expected protons are identified.

within the fiducial volume of the DDC. The mass resolution for $\Lambda$ 's is $4 \mathrm{MeV}$ and $11 \mathrm{MeV}$ for the $K_{s}^{0}$ 's. We expect to further increase the acceptance by identifying neutral decays occuring outside of the DDC.

\subsection{The DDC double $\Lambda$ results and $H_{0}$ predictions}

Double $\Lambda$ events are identified if two $\Lambda$ 's are reconstructed in the same event within $3 \sigma$ of the calculated invariant $\Lambda$ mass. From 15 million events we have identified 67 double $\Lambda$ events, this is consistent with the number of events expected from acceptance and efficiency calculations assuming $15 \Lambda$ 's produced per central collision. The increase in statistics from reconstructing the whole data set and extrapolating the neutral particle decay search to outside of the DDC should allow a significant sample to be obtained.

The $H_{0}$ reconstruction process is demonstrated in Fig.7 where a decaying $H_{0}$ has been embedded into a real DDC event. First the algorithm identifies a stiff positive particle, next a negative track is located with which the stiff positive track appears to form a secondary vertex within the active volume of the DDC. If successful, a search is then performed for a kink in the negative track. If all three steps are successful the event is flagged as containing a possible $H_{0}$ candidate and thus requiring further investigation. The $H_{0}$ embedded in Fig. 7 was correctly identified by this technique.

From calculations of the acceptance and efficiency for $H_{0}$ reconstruction using embedding of simulated $H_{0}$ 's into real data we can estimate our sensitivity for identifying $H_{0}$ 's. The sensitivity is strongly dependent on the lifetime of the $H_{0}$ and defined by

sensitivity $=\frac{\# H_{0}}{\# \text { events } \cdot a c c \cdot e f f \cdot B R}$ 


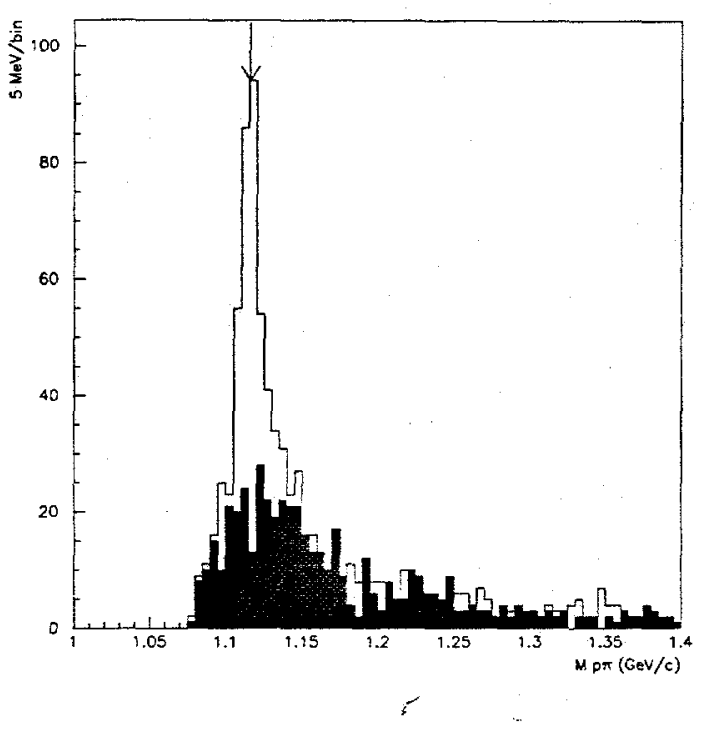

Figure 5. Invariant mass distribution for $\Lambda$ 's in the SDDA. 10,000 events have been reconstructed. See main text for details.
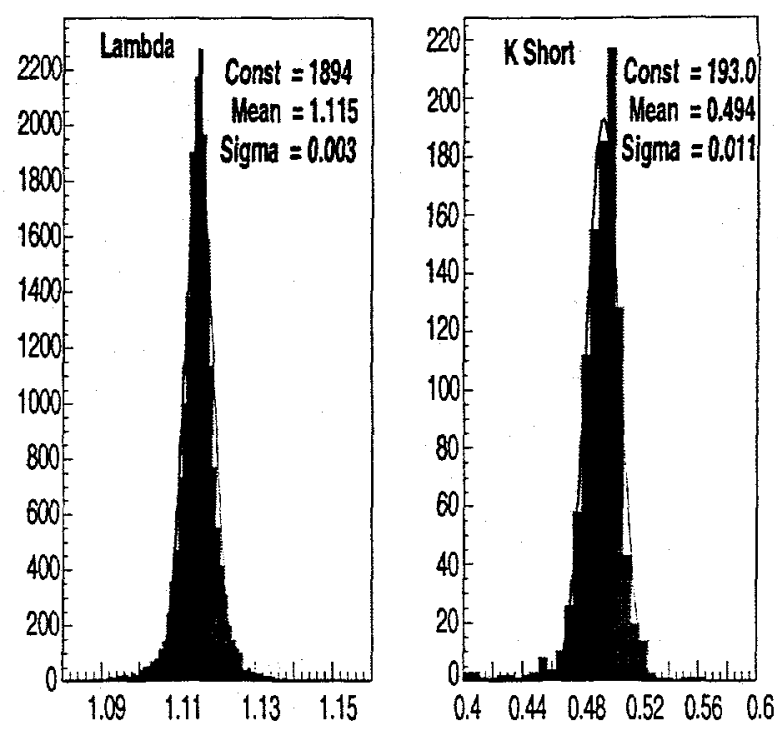

Figure 6. Invariant mass distributions of the $\Lambda$ and $K_{s}^{0}$ for the DDC. 15 million events have been reconstructed.

where the acceptance (acc) and efficiency (eff) were calculated from simulations, $10^{8}$ events were assumed and $2 H_{0}$ 's, with a branching ratio (BR) of $1 / 3$, were required to be identified to constitute a "discovery". The calculated sensitivity as a function of the $H_{0}$ lifetime is shown in Fig. 8.

\section{CONCLUSIONS}

The DDC recorded 100 million central events and the SDDA 680,000 central events. Based on the coalescence model, and assuming $0.1 \mathrm{H}_{0} /$ central collision and a $\mathrm{cr}=4 \mathrm{~cm}$, the DDC may reconstruct as many as $200 H_{0}$ 's. The statistics taken by both detectors should allow a definite statement to be made about the $H_{0}$ di-baryon existence.

The SDDA has been shown to be a mature technology able to track efficiently in a high multiplicity regime and high magnetic field. We are able to positively identify particles based on momentum and $d E / d x$. Given the number of events recorded by the SDDA during the run we expect to be able to identify a significant number of $\bar{p} s$. A preliminary $\Lambda$ invariant mass peak has aslo been reconstructed. With the expected improvement in reconstruction efficiency resulting from further calibration of the SDDA we expect to be able to measure the production of $\Lambda$ 's, $\bar{\Lambda}$ 's and $\Xi$ 's.

The DDC has identified $\Lambda$ 's and $K_{s}^{0}$ 's with a mass resolution of $4 \mathrm{MeV}$ and $11 \mathrm{MeV}$ respectively. The DDC has a good sensitivity for the $H_{0}$ and has currently identified 67 double $\Lambda$ events, when the whole data sample has been examined it is expected that a good statistical sample will have been identified. 


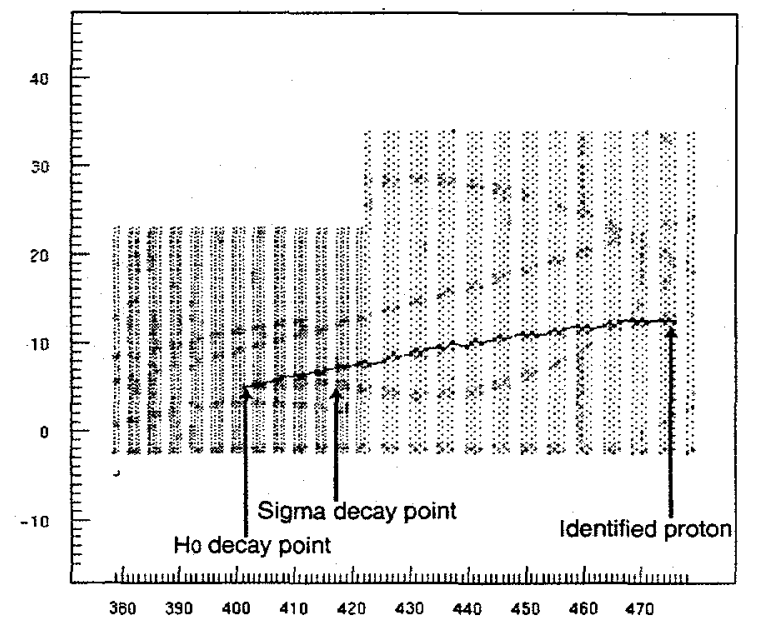

Figure 7. An embedded $H_{0}$ in a real DDC event and correctly reconstructed via the current software.

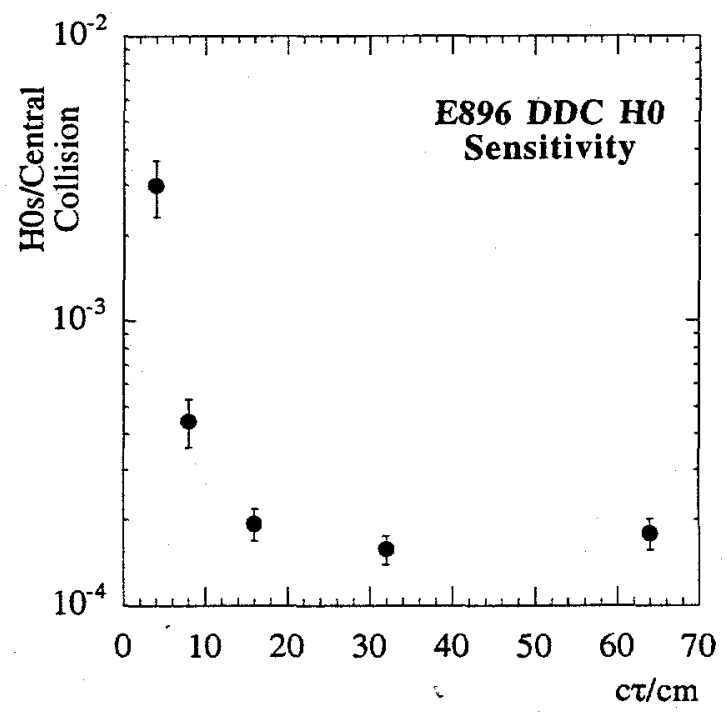

Figure 8. The DDC sensitivity to the $H_{0}$ as a function of lifetime.

\section{ACKNOWLEDGEMENTS}

Thanks to the whole E896 collaboration for all their help with this paper. I'd like to especially thank Tom Humanic, Gaspare Lo Curto, Jun Takahashi, Sean Kelly, Eleanor Judd, Rene Bellwied, Sanjeev Pandey and Hank Crawford for their particular help and input into the production of this document.

\section{REFERENCES}

1. R.L. Jaffe, Phys. Rev. Lett. 38 (1977) 195

2. R. Longacre et al., (E810 Collaboration), Nucl. Phys. A590 (1995) 477

3. J. Belz et al., (E888 Collaboration), Phys. Rev. Lett. 76 (1996) 3277

4. R.W. Stotzer et al., (E836 Collaboration), Phys. Rev. D28 (1997) 450

5. D. Ashery, AIP Conf. Proc. 432 (1998) 293

6. C.B. Dover et al., Phys. Rev. C40 (1989) 115

7. See parallel session on Strangeness in these proceedings for latest results.

8. See presentation by J. Nagle (E864 Collaboration) in these proceedings,

T.A. Armstrong et al., Phys. Rev. Lett. 79 (1997) 3351

9. G. Odyniec, Nucl. Phys. A638 (1998) 135c

10. E. Gatti and P. Rehak, Nucl. Instr. and Meth. A225 (1984) 608

11. J. Harris et al., (STAR Collaboration), Nucl. Phys. A566 (1994) 277

12. S. Albergo et al., Nucl. Instr. and Meth. A311 (1992) 280

13. G. Lo Curto parallel session report in these proceedings.

14. S.U. Pandey parallel session report in these proceedings. 


\title{
Memorandum of Understanding
}

\author{
Memorandum of Understanding Regarding Institutional Responsibilities for \\ Operation of the STAR Detector.
}

To achieve safe, effective, and efficient operation of the STAR Detector, it is agreed between the STAR Group at BNL and the participating STAR Collaborators at the University of California, Space Science Laboratory (hereafter referred to as "your group") that:

1) During the commissioning phase of STAR, and/or as necessary after prolonged shutdowns or upgrades, your group will maintain sufficient "expert" presence at BNL to bring the STAR trigger into full operation. Practical details (timing of visits, etc.) will be worked out with the STAR Operations Leader.

2) After routine operation of the STAR trigger has been established, further operation of this detector component will be the responsibility of STAR shift personnel who have been appropriately trained. Your group is responsible for developing any specialized training and/or documentation required to hand over routine operation of the trigger including written operating procedures as appropriate to STAR shift personnel.

3) After routine operation has been established, your group will not be expected to maintain an "expert" presence at BNL. However, your group will be expected to provide a call-down list which insures that someone with appropriate expertise may be reached at all times, with a delay of less than 1-2 hours. The decision to consult institutional experts will be made by the STAR Shift Leader or the STAR Operations Leader. Every reasonable attempt will be made to solve problems using on-site expertise before calling upon on-call experts. It will be the responsibility of your group to inform the STAR Operations Leader of any changes in the call-down list.

4) In the event of a serious problem, which can not be repaired with remote consultation, your group will be responsible for sending someone to $\mathrm{BNL}$ who has the expertise to fix the problem. An "Emergency Expert" should be able, if necessary, to travel to BNL with several days notice. The decision to send someone or not will be discussed with the STAR Operations Leader, and will be based on practical factors such as the severity of the problem, the uniqueness of the expertise which is required, and the availability of the component or system for repair on arrival of the expert.

5) Technical support for routine maintenance for contributed components or systems will be provided by the STAR Technical Support Group. Your group is responsible for developing any specialized training, documentation, and tools/hardware required to enable STAR technical support personnel to perform routine maintenance.

The undersigned parties agree to make every effort to honor this Memorandum of Understanding in the interest of achieving optimal performance of the STAR Detector. 
Signatures
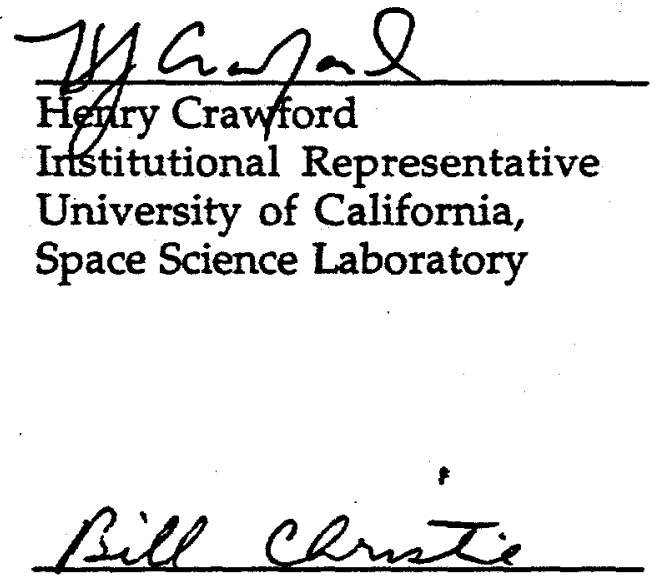

Bill Christie STAR Operations Leader

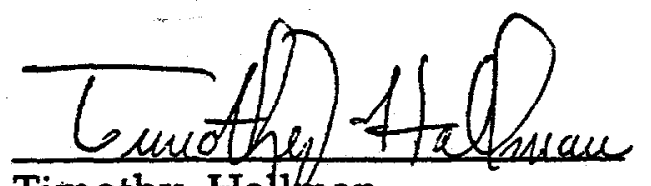

Timothy Hallman STAR Group Leader

Brookhaven National Laboratory
cc: J. Harris
J. Marx
S. Ozaki
D. Lowenstein
P. Pile

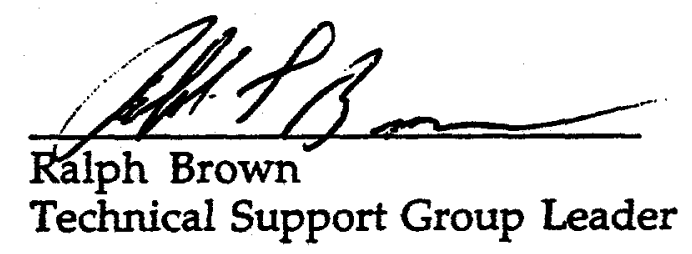


Electro Magnetic Calorimeter MOU No.

\title{
Memorandum of Understanding
}

\author{
between \\ University of California (Berkeley) Space Sciences Laboratory (UCB/SSL) \\ EMC Trigger Group \\ and \\ STAR EMC Collaboration \\ Project Management \\ at Wayne State
}

April 27, 1999

Draft

\section{Introduction}

This Memorandum of Understanding describes the collaboration by members of the University of California (Berkeley) Space Sciences Laboratory (UCB/SSL) in the STAR EMC. Note that there is already an existing MOU between UCB/SSL and STAR for the construction of the STAR baseline detector. The purpose of this collaboration is the design, fabrication, operation and scientific exploitation of the STAR EMC Detector, and upgrade to the baseline. The detector is described in the Technical Design Report, May 2, 1998, and subsequent technical documents elaborating that design.

This Memorandum of Understanding describes the anticipated funding from the DOE, together with the long-term contributions of UCB/SSL to the design, construction and operation of the STAR EMC Detector. It is understood that the anticipated contributions of UCB/SSL may later be modified or that additional responsibilities may be added to those described here.

This Memorandum of Understanding is made between UCB/SSL and STAR EMC Project Manager (PM). It does not constitute a legal contractual obligation on the part of either of the parties. It reflects an arrangement that is currently satisfactory to the parties involved. The parties agree to negotiate amendments to this memorandum as required to meet the evolving requirements of the STAR EMC research and development and detector construction program. 


\section{Personnel}

\subsection{List of Personnel}

Personnel committed to STAR EMC are expected to be:

\begin{tabular}{|l|l|l|l|}
\hline Name & $\begin{array}{l}\text { FY of } \\
\text { Activity }\end{array}$ & $\begin{array}{l}\text { EMC } \\
\% F T E\end{array}$ & Other Commitments \\
\hline E. Judd & FY99-00 & 25 & Trigger, E896 \\
\hline L. Greiner & FY99-00 & 25 & Trigger \\
\hline J. Engelage & FY99-00 & 20 & Trigger, E896 \\
\hline
\end{tabular}

\section{Design, Prototype, Production and Installation Responsibilities}

\subsection{Design, Prototype and Production Responsibilities - Construction Period}

\subsubsection{Project Description}

The UCB/SSL group will work with the Michigan State University group and other EMC collaborators to finalize details of the EMC trigger electronics requirements, agree on a final design for these electronics, fabricate all trigger related electronics, and operate the EMC trigger for STAR. For the purposes of STAR Trigger, the EMC is divided into 300 towers, each consisting of a number of photomultipliers which measure the light output from the colorimeter radiators. Our (UCB/SSL) task is to receive signals from these towers and determine whether an interesting event has occurred. We must therefore design both hardware to receive and store the digital tower signals and software to analyze these signals. We expect this task to begin in FY99 and to be completed in FY00.

The electronics design assumes that Trigger (TRG) will receive a bit stream from the EMC frontend-electronics (FEE) amounting to 12 bits per EMC tower, with 300 towers total. These will be routed to 30 Data-Storage-and-Manipulation (DSM) boards, with output from this first layer of DSMs feeding at least two more layers for a total of approximately 35 DSM boards. Each DSM board receives up to 128 bits of information each RHIC crossing, storing it for later retrieval and analyzing the bits in terms of the energy and spatial distributions they represent. Information from these towers is used in the level 0 trigger to find jets and correlated energy flow.

\subsubsection{Activities and Deliverables}

The overall STAR EMC activities project cost, without contingency and deliverables, are listed in the table below.

\begin{tabular}{|l|l|l|l|l|l|}
\hline WBS & $\begin{array}{l}\text { Project } \\
\text { Cost }\end{array}$ & FY & Deliverables & $\begin{array}{l}\text { Contributed } \\
\text { Labor }\end{array}$ & $\begin{array}{l}\text { Item Detail Including } \\
\text { Contributed Labor }\end{array}$ \\
\hline 4.5 .1 .12 .1 .1 & $0 \mathrm{k} \$$ & 99 & System Design & $1 \mathrm{MMP}$ & $1.5 \mathrm{MM} \mathrm{EE}$ \\
\hline 4.5 .1 .12 .1 .2 & $105 \mathrm{k} \$$ & 00 & 35 DSM Boards & $2 \mathrm{MMP}$ & $\begin{array}{l}\text { Fab and test 35 DSM boards for } \\
\text { L0 }\end{array}$ \\
\hline
\end{tabular}




\begin{tabular}{|l|l|l|l|l|l|}
\hline 4.5 .1 .12 .1 .3 & $30 \mathrm{k} \$$ & 00 & $\begin{array}{l}\text { 2 VME Crates, } \\
\text { CPUs, SCI Nodes }\end{array}$ & & Infrastructure for L0 \\
\hline 4.5 .1 .12 .1 .4 & $10 \mathrm{k} \$$ & 00 & $\begin{array}{l}\text { Cables and Misc. } \\
\text { Hardware }\end{array}$ & & \\
\hline 4.5 .1 .12 .1 .5 & $15 \mathrm{k} \$$ & 00 & $\begin{array}{l}\text { Installation and } \\
\text { Test }\end{array}$ & $2 \mathrm{MM} \mathrm{P}$ & Install L0 hardware at STAR \\
\hline 4.5 .1 .12 .1 .6 & $0 \mathrm{k} \$$ & $\begin{array}{l}99 \\
-\end{array}$ & $\begin{array}{l}\text { Software for Data } \\
\text { Selection }\end{array}$ & $2 \mathrm{MM} \mathrm{P}$ & \\
\hline
\end{tabular}

The total cost is $160 \mathrm{k} \$$. Determination of the allocation of project funds to the subsystems of the STAR EMC is the responsibility of STAR EMC subsystem manager (Cormier). Allocations are determined each FY based on the overall project budget and the priorities needs within the EMC project.

\subsection{Coordination and Reporting}

The STAR EMC Subsystem Manager for the Calorimeter subsystem is Tom Cormier. The institution contact person for Calorimeter electronic activities at UCB/SSL is Hank Crawford. The taskmaster at UCB/SSL is Leo Greiner. The Manager for the STAR EMC electronics is Richard Jared.

The progress of the design, fabrication, and testing of these components will be reported by the institute taskmaster on a monthly basis, by WBS element to L4 in detail (cost and progress), to STAR EMC electronics manger with concurrent copy to the institute contact. The STAR EMC electronic manger will report subsystem progress to EMC subsystem manager. The EMC subsystem manager will report to STAR management.

\section{Contribution of Effort, Services and Facilities}

\subsection{Effort}

Subject to adequate base program funding by DOE, UCB/SSL will provide support for technical personnel as indicated below.

\begin{tabular}{|l|l|l|}
\hline Name & FY99 & FY00 \\
\hline E. Judd & $2 \mathrm{MM}$ & $2 \mathrm{MM}$ \\
\hline J. Engelage & $2 \mathrm{MM}$ & $2 \mathrm{MM}$ \\
\hline
\end{tabular}

\subsection{Operating Costs}

UCB/SSL, subject to adequate base program funding from DOE, will support the normal research operating expenses (such as physicists' salaries, travel expenses, miscellaneous supplies, administrative support, etc.) of the UCB/SSL group working on the STAR EMC project. These normal operating expenses are not considered as part of the STAR EMC detector cost estimate.

\section{Administration}




\subsection{Method of Funding Transfers and Purchasing}

The expenditures by UCB/SSL are to be covered by funds provided by DOE, upon the allocation decision of the STAR EMC project management and from funds provided from UCB/SSL base research program. Project Funds to cover work described in this document will be provided directly to UCB/SSL by RHIC.

\section{Schedules and Milestones}

UCB/SSL will make every effort to carry out their institutional responsibilities consistent with the schedules for the fabrication of the STAR EMC detector. These schedules may have to be changed as the project progresses. Changes that affect UCB/SSL will be noted in Amendments to this Memorandum.

\subsection{Design, Fabrication and Installation Milestones}

The key milestones relevant to UCB/SSL are listed here:

\begin{tabular}{|l|l|l|}
\hline WBS & Milestone & $\begin{array}{l}\text { Baseline } \\
\text { Date }\end{array}$ \\
\hline 1.1 .11 .2 & First DSM boards & $11 / 99$ \\
\hline 1.1 .11 .3 & Infrastructure installed & $11 / 99$ \\
\hline & & \\
\hline
\end{tabular}

\section{Makers and Concurrence}

The following persons concur in the terms of this Memorandum of Understanding. These terms will be updated as appropriate in Amendments to this Memorandum.

Makers of this Memorandum:

\begin{tabular}{l}
\hline Jay Marx \\
STAR Project Manager
\end{tabular}

Richard Jared

EMC Electronics Manager
Tom Cormier date

EMC Subsystem Manager

Wayne State date

Hank J. Crawford Institution Representative

UCB/SSL 